\title{
Producción de Leche de Suiz-Bú, una Raza de Doble Propósito en el Trópico Mexicano
}

\author{
Milk Production of Suiz-Bu, a Dual Purpose Cattle Breed in the Mexican Tropics \\ Nicacia Hernández-Hernández ${ }^{1}$, Juan Carlos Martínez-González ${ }^{1,3}$, \\ Gaspar Manuel Parra-Bracamonte ${ }^{2}$, Arnoldo González-Reyna ${ }^{1}$, \\ Eugenia Guadalupe Cienfuegos-Rivas ${ }^{1}$, Javier Hernández-Meléndez ${ }^{1}$
}

\section{Resumen}

El objetivo del presente trabajo fue determinar el efecto de factores ambientales sobre la producción de leche de vacas Suiz-Bú en las condiciones del trópico mexicano. Se analizaron los registros de producción de leche de 728 vacas con información disponible de los años 2012 al 2014. Las vacas se ordeñaban dos veces al día a partir del quinto día posparto y eran alimentadas bajo condiciones de pastoreo y suplementación con concentrado comercial. Las variables producción de leche a 305 días (PL305) y media de producción diaria (MPD) fueron analizadas con un modelo mixto que incluyó efectos de número de lactancia (NL), año de parto (AP), época de parto (EP) y las interacciones simples. El año de parto fue estadísticamente significativo para PL305 y PMD $(\mathrm{p}<0.05)$, las vacas que parieron en la época lluviosa tuvieron una mayor producción, tanto PL305 como PMD, que aquellas que parieron en época seca $(\mathrm{p}<0.05)$, y las vacas de primer parto tuvieron una menor PL305 que vacas con dos o tres lactancias $(p<0.05)$. Asimismo, los efectos de las interacciones fueron significativas $(\mathrm{p}<0.05)$.

Palabras clave: lactancia, B. indicus x B. taurus, recursos naturales, trópico

\section{Abstract}

The aim of this study was to determine the effect of environmental factors on milk production of Suiz-Bu cows reared under the Mexican tropical conditions. Milk yield records of 728 cows that were available from 2012 to 2014 were analyzed. Cows were

${ }^{1}$ Centro Universitario Adolfo López Mateos, Facultad de Ingeniería y Ciencias, Universidad Autónoma de Tamaulipas, Ciudad Victoria, Tamaulipas, México

${ }^{2}$ Laboratorio de Biotecnología Animal, Centro de Biotecnología Genómica, Instituto Politécnico Nacional, Reynosa, Tamaulipas, México

${ }^{3}$ E-mail: jmartinez@docentes.uat.edu.mx

Recibido: 2 de febrero de 2016

Aceptado para publicación: 2 de agosto de 2016 
milked twice a day as of the fifth day of calving, fed under grazing conditions and supplemented with commercial concentrates. Variables of milk production at 305 days (PL305) and average milk yield per day (MPD) were analyzed using a mixed model that included effects of lactation (NL), year of calving (AP), season of calving (EP), and simple interactions. The year of calving was statistically significant for PL305 and PMD $(p<0.05)$, cows that calved during the rainy season had greater milk production (PL305 and PMD) than those calving during the dry season $(\mathrm{p}<0.05)$, and first calvers had lower PL305 than cows with two or three lactations $(p<0.05)$. Moreover, the effects of interactions were significant $(\mathrm{p}<0.05)$.

Key words: lactation, $B$. indicus $\mathrm{x}$ B. taurus, natural resources, tropics

\section{INTRODUCCIÓN}

El incremento de la población en el ámbito nacional y mundial tiene como consecuencia un aumento en la demanda de productos pecuarios (leche y carne), por lo que es necesario buscar alternativas para incrementar el volumen de estos productos (ÁngelesMontiel et al., 2004; FAO, 2015). Una alternativa es utilizar el sistema de producción con bovinos de doble propósito mediante el cruzamientos de razas especializadas $B$. taurus con $B$. indicus para obtener animales de doble propósito (leche y carne) (Chan et al., 2010; Ríos-Utrera et al., 2015).

El reconocimiento, en México, de la raza Suiz-bú data de la década de los ' 80 . Esta raza se generó con el fin de obtener un animal apto para las condiciones del trópico y de producción eficiente, contemplando características como docilidad, longevidad, rusticidad, precocidad, conversión (rápido desarrollo), fertilidad, facilidad de parto, habilidad materna, marcada pigmentación, así como adecuada producción de leche (rica en grasa) y carne (AMCSB, 2016).

El sistema de ganado de doble propósito es considerado como un sistema ganadero tradicional de producción de la región tropical, donde las vacas son ordeñadas una o dos veces al día con apoyo de la cría y su principal fuente de alimentación es a base de pastoreo (Hernández-Reyes et al., 2000).
La producción animal en el trópico representa una de las alternativas más importantes que tiene México para disminuir el déficit de leche (Carvajal-Hernández et al., 2002), debido al número de vacas que se dispone y por la cantidad de recursos naturales utilizables básicos, como suelo, agua y pasto, que contribuyen a una producción de bajo costo en comparación con el uso de recursos externos como concentrados (Magaña et al., 2006). Sin embargo, el mejoramiento de la producción de leche en condiciones tropicales depende de factores directos relacionados con el animal (Román-Ponce et al., 2013) y el genotipo (Magaña et al., 2009; Cabrera et al., 2013), así como factores ambientales como alimentación, manejo, días en lactancia y número de parto (Pollott, 2000; CarvajalHernández et al., 2002; Jílek et al., 2006; Yano et al., 2014).

En este sistema de producción, bajo las condiciones de México, no existen datos productivos por vaca, lo que crea la necesidad de promover registros de producción para detectar las diferencias en el potencial productivo de los animales (García-Muñiz et al., 2007; Magaña et al., 2009). Es así que se requiere que las unidades de producción utilicen métodos para organizar la información que se genera a diario, de modo que permita evaluar el comportamiento productivo de los animales (Zambrano y Contreras, 2005). Una forma de evaluar el comportamiento productivo es mediante la evaluación de las características de producción de leche (López et 
al., 2009; López-Ordaz et al., 2009), de allí que el objetivo del presente trabajo fue evaluar el efecto de factores ambientales sobre la producción de leche en vacas Suiz-Bú en condiciones tropicales.

\section{Materiales y Métodos}

El estudio fue realizado con datos de animales registrados en la Asociación Mexicana de Criadores de Ganado Suiz-Bú de Registro A.C. (AMCSB), con sede en Tampico, Tamaulipas, México. Se analizaron los registros de producción de leche de 728 vacas con información disponible del periodo 2012 a 2014. Todas las unidades de producción estaban ubicadas en las regiones tropicales de México.

La alimentación del ganado estaba basada en el pastoreo rotacional con gramas nativas (Axonopus sp y Paspalum sp) y pastos introducidos como estrella de África (Cynodon plectostachyus) y Guinea (Panicum maximum). Además, las vacas recibieron una suplementación de concentrado comercial a la hora de la ordeña. El ordeño de las vacas se inició a partir del quinto día posterior al parto, dos veces al día y con apoyo del becerro. Las producciones de leche eran registradas de forma individual en la base de datos de la AMCSB.

Se analizó la producción de leche a 305 días (PL305), definida como los kilogramos de leche producidos por vaca durante la lactancia, y la media de producción de leche por día (MPD). Para homogenizar los días en lactancia se realizaron ajustes multiplicativos, de acuerdo a lo sugerido por Wood (1967).

Los datos fueron analizados con un modelo mixto que incluyó el efecto fijo del número de lactancia $(\mathrm{NL}=1,2,3)$, año de parto (AP $=2012,2013,2014)$, época de parto $(\mathrm{EP}=$ seca [enero-junio], lluviosa [julio-diciembre]) y las interacciones simples
$(\mathrm{NL} \times \mathrm{AP}, \mathrm{NL} \times \mathrm{EP}$ y $\mathrm{AP} \times \mathrm{EP}) . \mathrm{El}$ modelo fue analizado con el procedimiento MIXED del paquete estadístico SAS (SAS, 2004) y definido como:

$\mathrm{Y}_{\mathrm{ijk} k}=\mu+\mathrm{NL}_{\mathrm{i}}+\mathrm{AP}_{\mathrm{j}}+\mathrm{EP}_{\mathrm{k}}+(\mathrm{NL} \times \mathrm{AP})_{\mathrm{ij}}+$
$(\mathrm{NL} \times \mathrm{EP})_{\mathrm{ik}}+(\mathrm{AP} \times \mathrm{EP})_{\mathrm{jk}}+\mathrm{E}_{\mathrm{ijk}}$

donde:

$\mathrm{Y}_{\mathrm{ijk} \mathrm{l}}=$ Es la variable PL305 y MPD; $\mu=$ Media general del PL305 y MPD; $\mathrm{NL}_{\mathrm{i}}=$ Efecto fijo del i-ésimo número de lactancia; $\mathrm{AP}_{\mathrm{j}}=$ Efecto fijo del j-ésimo año de parto; $\mathrm{EP}_{\mathrm{k}}{ }_{\mathrm{k}}=$ Efecto fijo de la k-ésima época de parto; $(\mathrm{NL} \times \mathrm{AP})_{\mathrm{ij}}=$ Efecto de la interacción número de lactancia $x$ año de parto; $(\mathrm{NL} \times \mathrm{EP})_{\mathrm{ik}}=$ Efecto de la interacción número de lactancia $\mathrm{x}$ época de parto; $(\mathrm{AP} \times \mathrm{EP})_{\mathrm{jk}}=$ Efecto de la interacción año de parto $\mathrm{x}$ época de parto; $\mathrm{y}$ $\mathrm{E}_{\mathrm{ijk} \mathrm{l}}=$ Error aleatorio.

Las variables que tuvieron efecto significativo fueron analizadas con la prueba de comparación de medias de Tukey $(\mathrm{p}<0.05)$.

\section{Resultados y Discusión}

\section{Producción de Leche}

La producción media de leche para PL305 y MPD fue de 4328.1 y $14.2 \mathrm{~kg}$, respectivamente. Estos datos son ajustados a 305 días de lactancia; no obstante, la duración real de la lactancia fue de $180 \pm 20$ días. Estos resultados son menores que los reportados por Vite-Cristóbal et al. (2007), quienes realizaron un estudio con ganado de doble propósito alimentado con forrajes tropicales y concentrado, obteniendo una media para la tercera lactancia de $4552 \mathrm{~kg}$ de leche ajustada a 270 días y $14.1 \mathrm{~kg}$ leche por día de lactancia. Contrario a este resultado, RíosUtrera et al. (2015) en vacas Simmental $\times$ Cebú y Suizo Pardo $\times$ Cebú, alimentadas con forrajes y concentrado, obtuvieron una media de $1766 \mathrm{~kg}$ de leche por lactancia de 260 días. 
Estas diferencias pueden ser atribuidas a las diferentes condiciones de alimentación y manejo de los animales. Por ejemplo, el número de días en lactancia que se manejan en zonas tropicales es de 120 a 270 días (ViteCristóbal et al., 2007; Salamanca y Bentez, 2012; AMCSB, 2016).

\section{Año de Parto}

Las medias de PL305 y MPD fueron significativamente menores en 2012 que en los años siguientes (Cuadro 1). Las diferencias en las condiciones climáticas, que inciden además con la calidad y cantidad de las pasturas, afectan significativamente los niveles productivos entre años (Carvajal-Hernández et al., 2002; Ríos-Utrera et al., 2015). En este sentido, Hernández-Reyes et al. (2000), ViteCristóbal et al. (2007) y López et al. (2009) indican que el año de parto es un factor difícil de explicar debido a que incluye la variación de componentes ambientales, la disponibilidad de pastos, potreros, carga animal, y los cambios en el manejo del hato.

\section{Época de Parto}

La época de parto tuvo efecto significativo sobre la PL305 y a la MPD $(\mathrm{p}=0.0003)$. Las vacas que parieron en época lluviosa tuvieron mayor producción (PL305=4409.1 kg; $\mathrm{MPD}=14.5 \mathrm{~kg}$ ) en comparación con las vacas con partos en la época seca (PL305=4238.2 kg; MPD=13.9 kg) (Cuadro 1). En forma similar, García-Muñiz et al. (2007), trabajando con vacas Holstein en agroempresas familiares, reportaron una mayor producción de leche en vacas con partos en época lluviosa. Estas diferencias pueden ser atribuidas a la menor cantidad y calidad del forraje que se presenta en la época seca (Vásquez et al., 2012), y posiblemente a que los niveles de suplementación no cubrían las necesidades de producción.

Por otra parte, Ríos-Utrera et al. (2015), trabajando con vacas Simmental x cebú y Suizo Pardo x cebú, no encontraron diferen- cias en producción de leche entre las vacas que parieron en la época fría y lluviosa. Asimismo, Cabrera et al. (2013) tampoco encontraron un efecto de la época de parto sobre la producción de leche en vacas de doble propósito. En estos casos, los resultados se pueden explicar por la adición de concentrados que suministran nutrientes adicionales y suficientes que evitan las deficiencias nutrimentales en la época seca, permitiendo obtener producciones de leche semejantes en ambas épocas.

\section{Número de Lactancia}

No se encontraron diferencias significativas en MPD, pero si hubo para PL305 $(\mathrm{p}=0.0001)$, donde las vacas de primera lactancia produjeron menor cantidad de leche (Cuadro 1). En forma similar, y como es esperado, García-Muñiz et al. (2007), en su investigación con vacas Holstein en agroempresas familiares, y Hernández-Reyes et al. (2000) con vacas de doble propósito, indicaron que las vacas de primera lactancia presentaron las menores producciones de leche comparadas a vacas de dos o más lactancias. No obstante, Aranguren-Méndez et al. (1994), trabajando con vacas mestizas $5 / 8$ Holstein, 5/8 Pardo Suizo y 5/8 Brahman en las condiciones tropicales de Venezuela, no encontraron diferencias en producción de leche entre lactancias.

Las diferencias entre las producciones de leche según el número de lactancia se deben al ajuste fisiológico y endocrinológico de las vacas de primer parto, así como su continuo desarrollo corporal para alcanzar la talla y el peso de una vaca adulta (HernándezReyes et al., 2000; Pollott, 2000). En un estudio realizado por Bormann et al. (2002), comprobaron que vacas que paren a una corta edad tienen una menor producción de leche durante su primera lactancia; sin embargo, el rendimiento durante su vida productiva es significativamente mayor que en aquellos animales que tuvieron su primer parto a una edad más avanzada. 
Cuadro 1. Medias de cuadrados mínimos y error estándar para producción de leche a 305 días (PL305) y media de producción de leche por día (MPD) de acuerdo al año de parto, época de parto y número de lactancia de vacas Suiz-Bú en condiciones tropicales

\begin{tabular}{|c|c|c|c|}
\hline & $\mathrm{n}$ & PL305 & MPD \\
\hline \multicolumn{4}{|l|}{ Año de parto } \\
\hline 2012 & 146 & $4046.9 \pm 1196.3^{b}$ & $13.3 \pm 3.9^{b}$ \\
\hline 2013 & 315 & $4406.9 \pm 806.5^{\mathrm{a}}$ & $14.5 \pm 2.6^{\mathrm{a}}$ \\
\hline 2014 & 267 & $4388.9 \pm 924.2^{\mathrm{a}}$ & $14.4 \pm 3.0^{\mathrm{a}}$ \\
\hline \multicolumn{4}{|l|}{ Época de parto } \\
\hline Lluviosa & 383 & $4409.1 \pm 944.6^{\mathrm{a}}$ & $14.5 \pm 3.1^{\mathrm{a}}$ \\
\hline Seca & 345 & $4238.2 \pm 945.1^{\mathrm{b}}$ & $13.9 \pm 3.1^{b}$ \\
\hline \multicolumn{4}{|c|}{ Número de lactancia } \\
\hline 1 & 425 & $4191.6 \pm 969.8^{b}$ & $13.7 \pm 3.2^{\mathrm{a}}$ \\
\hline 2 & 282 & $4517.4 \pm 855.4^{\mathrm{a}}$ & $14.8 \pm 2.8^{\mathrm{a}}$ \\
\hline 3 & 21 & $4548.7 \pm 1224.7^{\mathrm{a}}$ & $14.9 \pm 4.0^{\mathrm{a}}$ \\
\hline
\end{tabular}

\section{Interacciones}

Las interacciones simples $\mathrm{NL} \times \mathrm{AP}$, $\mathrm{NL} \times \mathrm{EP}$ y $\mathrm{AP} \times \mathrm{EP}$ para las variables PL305 y MPD fueron significativas $(\mathrm{p}<0.05)$. En la interacción año de parto por época de parto para las variables PL305 y MPD, se observó que las vacas que parieron tanto en la época seca como en la lluviosa en los años 2013 y 2014 tuvieron mayor producción de leche en comparación con el año 2012 en la época lluviosa, donde su producción fue menor. Este es un claro efecto de los cambios climáticos y de manejo de los animales, tanto a través de los años como de las épocas. Estos resultados son similares a los reportados por diversos autores (Ribas et al., 2004; Fernández y Tronco, 2011).

En la interacción número de lactancia por época de parto, se observó que las vacas produjeron más leche en la tercera lactancia y la época lluviosa en comparación con la época seca.

\section{Conclusión}

Factores ambientales como año y época de parto, así como el número de lactancia tienen un efecto significativo en la producción de leche de las vacas Suiz-Bú criadas en el trópico mexicano.

\section{Agradecimiento}

Los autores agradecen a la Asociación Mexicana de Criadores de Ganado Suiz-Bú de Registro A. C. por las facilidades prestadas para la realización del presente estudio.

\section{Literatura Citada}

1. [AMCSB] Asociación Mexicana de Criadores de Ganado Suiz-Bú de Registro A.C. 2013. Reglamento técnico [Internet]. Disponible en: http://www. suizbu.com.mx/ver_menus.-php? opcion $=$ ver\&id_padre $=\& \mathrm{id}=835$ 
2. Ángeles-Montiel R, Mora-Flores JS, Martínez-Damián MA, García-Mata R. 2004. Efecto de las importaciones de leche en el mercado nacional del producto. Agrociencia 38: 555-564.

3. Aranguren-Méndez JA, GonzálezStagnaro C, Madrid-Bury N, Ríos J. 1994. Comportamiento productivo de vacas mestizas 5/8 Holstein, 5/8 Pardo Suizo y 5/8 Brahman. Rev Cient FCVLUZ 4: 99-106.

4. Bormann J, Wiggans GR, Druet T, Gengler N. 2002. Estimating effects of permanent environment, lactation stage, age and pregnancy on test-day yield. J Dairy Sci 85: 263-284. doi: 10.3168/ jds.S0022-0302(02)74076-9

5. Cabrera NA, Lammoglia VMA, Daniel RIC, Elorza MP. 2013. Efecto del genotipo sobre la producción láctea en vacas Europeo Lechero x Cebú de doble propósito. Rev Biol Agropec Tuxpan 1: 51-57.

6. Carvajal-Hernández $M$, ValenciaHeredia ER, Segura-Correa JC. 2002. Duración de la lactancia y producción de leche de vacas Holstein en el Estado de Yucatán, México. Rev Biomed 13: 25-31.

7. Chan EKF, Nagaraj SH, Reverter A. 2010. The evolution of tropical adaptation: comparing taurine and zebu cattle. Anim Genet 41: 467-477. doi: 10.1111/j.1365-2052.2010.02053.x

8. [FAO] Organización de las Naciones Unidas para la Alimentación y la Agricultura. 2015. Producción lechera. [Internet]. Disponible en: http:// www.fao.org/agriculture/dairy-gateway/ milk-production/en/\#.VhLMJeyqqko

9. Fernández FJ, Tronco MAS. 2011. Influencia de factores no genéticos en la producción de leche del Siboney de Cuba. Rev Salud Anim 33: 76-82.

10. García-Muñiz JG, Mariscal-Aguayo DV, Caldera-Navarrete NA, RamírezValverde $R$, Estrella-Quintero $H$, Núñez-Domínguez, R. 2007. Variables relacionadas con la producción de leche de ganado Holstein en agroempresas familiares con diferente nivel tecnológico. INCI 32: 841-846.

11. Haworth G, Tranter W, Chuck J, Cheng Z, Wathes V. 2008. Relationships between age at first calving and first lactation milk yield, and lifetime productivity and longevity in dairy cows. Vet Rec 162: 643-647. doi: 10.1136/ vr.162.20.643

12. Hernández-Reyes E, Segura-Correa VM, Segura-Correa JC, Osorio-Arce MM. 2000. Intervalo entre partos, duración de la lactancia y producción de leche en un hato de doble propósito en Yucatán, México. Agrociencia 34: 699705 .

13. Jílek F, Øehák D, Volek J, Štípková $M$, Nimcová E, Fiedlerová $M$, et al. 2006. Effect of herd, parity, stage of lactation and milk yield on urea concentration in milk. Czech J Anim Sci 51:510-517.

14. López OR, García RC, García JGM, Ramírez RV. 2009. Producción de leche de vacas con diferente porcentaje de genes Bos taurus en el trópico mexicano. Téc Pecu Méx 47: 435-448.

15. López-Ordaz R, Vite-Cristóbal C, García-Muñiz JG, MartínezHernández PA. 2009. Reproducción y producción de leche de vacas con distinta proporción de genes Bos taurus. Arch Zootec 58: 683-694.

16. Magaña JG, Parra BGM, Estrada LJR, Kú VJC, Sosa FCF. 2009. Caracterización del recurso genético animal en el diseño de sistemas sustentables de producción bovina en el trópico. Trop Subtrop Agroeco 10: 85-94.

17. Magaña MJG, Ríos GA, Martínez JCG 2006. Los sistemas de doble propósito y los desafíos en los climas tropicales de México. Arch Latinoam Prod Anim 14: 105-114.

18. Pollott GE. 2000. A biological approach to lactation curve analysis for milk yield. J Dairy Sci 83: 2448-2458. doi: 10.3168/ jds.S0022-0302(00)75136-8 
19. Ribas M, Gutiérrez M, Mora M, Évora JC, González S. 2004. Comportamiento productivo y reproductivo del Siboney de Cuba en dos localidades. Rev Cub Cienc Agríc 38: 121-124.

20. Ríos-Utrera Á, Hernández-Hernández VD, Amezcua-Manjarréz EV, Zárate-Martínez JP. 2015. Producción láctea de vacas Simmental x cebú y Suizo Pardo x cebú en clima tropical. Agron Mesoam 26: 17-25. doi: 10.15517/ am.v26i1.16891

21. Román-Ponce SI, Ruiz-López FJ, Montaldo HH, Rizzi R, Román-Ponce H. 2013. Efectos de cruzamiento para producción de leche y características de crecimiento en bovinos de doble propósito en el trópico húmedo. Rev Mex Cienc Pecu 4: 405-416.

22. Salamanca CA, Bentez MJ. 2012. Producción de leche de vacas mestizas del sistema doble propósito en el municipio de Arauca. REDVET 13(7). [Internet]. Disponible en: http://www.redalyc.org/ articulo.oa? $\mathrm{id}=63624404008$

23. SAS. 2004. The SAS 9.1 for Windows. SAS Institute Inc. Cary, North Carolina. USA.
24. Vásquez F, Danilo P, Mora-Delgado J, Skarpe C. 2012. Selectividad de especies forrajeras por bovinos en pastizales seminaturales del trópico centroamericano: un estudio basado en la observación sistemática del pastoreo. Zootecnia Trop 30: 63-80.

25. Vite-Cristóbal C, López-Ordaz $R$, García-Muñiz JG, Ramírez-Valverde R, Ruiz-Flores A, López-Ordaz R. 2007. Producción de leche y comportamiento reproductivo de vacas de doble propósito que consumen forrajes tropicales y concentrados. Vet Méx 38: 63-79.

26. Wood PDP. 1967. Algebraic model of the lactation curve in cattle. Nature 216: 164-165. doi: 10.1038/216164a 0

27. Yano M, Shimadzu H, Endo T. 2014. Modelling temperature effects on milk production: a study on Holstein cows at a Japanese farm. Springerplus 3: 1-11. doi: 10.1186/2193-1801-3-129

28. Zambrano SRA, Contreras R. 2005. Estimación de valores reales de producción a partir de pesajes alternos en 3 rebaños bovinos lecheros. Arch Latinoam Prod Anim 13: 215. [Internet]. Disponible en: http://www.bioline.org.br/ pdf?la05022 